PROSTATE CANCER

\section{Circulating free DNA as biomarker}

Genomic analysis of serial, circulating free DNA (cfDNA) samples in patients undergoing PARP inhibitor (PARPi) therapy can be used to predict treatment responses and monitor emergence of PARPi resistance mutations, according to a new study in Cancer Discovery.

The TOPARP-A trial had found that the PARPi olaparib had antitumour activity in men with metastatic castration-resistant prostate cancer, which was associated with mutations in homologous DNA recombination (HDR) genes. During the trial, the investigators had collected cfDNA samples to test their ability to predict response and elucidate the molecular evolution of PARPi resistance.

$A \geq 50 \%$ decrease in cfDNA concentration at week 8 of treatment was associated with extended overall survival in responders (HR 0.19, 95\% Cl 0.06-0.56; $P=0.003$ ) in multivariable analysis. All somatic HDR-associated mutations in tumour tissues were detectable in cfDNA samples. Targeted sequencing showed that the allele frequency of somatic mutations selectively decreased in responders $\left(\chi^{2} P<0.001\right)$. In five responders of six patients who had somatic mutations related to HDR deficiency in their tumours, the somatic mutation allele frequency decreased to $<5 \%$. Investigating germline mutations in BRCA2 or ATM, the team found that in four of five responding patients who had loss of heterozygosity for these mutations their allele frequency decreased to $\sim 50-60 \%$.

Analysis of cfDNA samples from the time of resistance development and disease progression showed that in two patients who had germline and one patient who had a somatic BRCA2 frameshift mutation, as well as one patient with a somatic PALB2 mutation, additional new somatic mutations restored the normal open reading frame. The data suggest the emergence of divergent PARPi-resistant tumour subclones that are functionally convergent, driven by selective therapeutic pressure.

These findings indicate that the use of cfDNA analysis as a simple liquid biopsy approach could enable enhanced personalized patient management, improving outcomes and reducing overtreatment and costs.

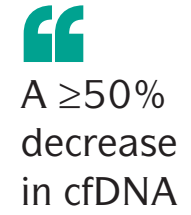

concentration

... was

associated

with extended

overall survival

in responders

Clemens Thoma 